\title{
In Situ TEM Studies of Nanoparticle Growth in a Fluorozirconate (ZBLAN) Glass Matrix
}

\author{
M. Vu*, C. Alvarez**, Y. Liu***, A. K. Petford-Long****, J. A. Johnson* \\ * University of Tennessee, Materials Science and Engineering Dept, Tullahoma TN 37388 \\ ** Materials Science and Engineering, Northwestern University, Evanston IL 60208 \\ *** Materials Science Division, Argonne National Laboratory, Argonne IL 60439 \\ $* * * *$ Center for Nanoscale Materials, Argonne National Laboratory, Argonne IL 60439 \\ ZBLAN (Zirconium, Barium, Lanthanum, Aluminum, and Sodium Fluorides) glass-ceramic \\ materials are being developed as an imaging plate mainly for medical applications [1]. The \\ materials are doped with europium and chlorine and can be heat treated in such a way that they \\ form a novel nanocomposite material containing barium chloride nanocrystals, with the ability to \\ convert ionizing radiation (usually x-rays) into stable electron-hole pairs. The image can be read \\ out afterwards with a scanning laser beam in a so-called "photostimulated luminescence" (PSL) \\ process.
}

The ZBLAN glass only acts as an imaging plate upon annealing. As the annealing temperature and annealing time are increased, so a higher degree of nucleation of $\mathrm{BaCl}_{2}$ crystallites inside the glass matrix is observed. As a result, more crystallites are available to incorporate $\mathrm{Eu}^{2+}$ and hence increase the fluorescence intensity. However, a higher annealing temperature and a longer annealing time also lead to a larger degree of crystal growth, resulting in bigger nanoparticles. This leads to a decrease in spatial resolution of a ceramic-glass storage phosphor. The optimal annealing condition thus needs to compromise between the fluorescence intensity and the spatial resolution.

In this Digest we report on the results of ex situ and in situ TEM studies of ZBLAN glasses, being carried out to further understand the growth of nanoparticles inside a glass matrix under various heating conditions.Plan-view samples of the glasses were prepared by grinding/polishing followed by ion-beam milling at liquid nitrogen temperature to prevent crystallization during sample preparation.

Figure 1 shows a low magnification dark field TEM image of a sample prepared from an aspoured modified ZBLAN glass, together with a selected area diffraction pattern. The lack of contrast in the dark field image and the broad rings in the diffraction pattern show that the region is amorphous. No evidence for any crystalline contrast was observed across the whole sample. By comparison, Figure 2 shows a bright field image and corresponding electron diffraction pattern of the same area after the sample was annealed in the TEM. The contrast of the small regions and the polycrystalline diffraction indicate nanocrystal formation during annealing; the size of the formed nanocrystals however, varies from a few nanometers to tens of nanometers. Figure 3 shows initial in situ HREM images with different sizes of $\mathrm{BaCl}_{2}$ nanocrystals. The structural and compositional studies of as-synthesized and ex situ heat-treated material and in situ TEM heating studies to following nanocrystal formation will be presented. 


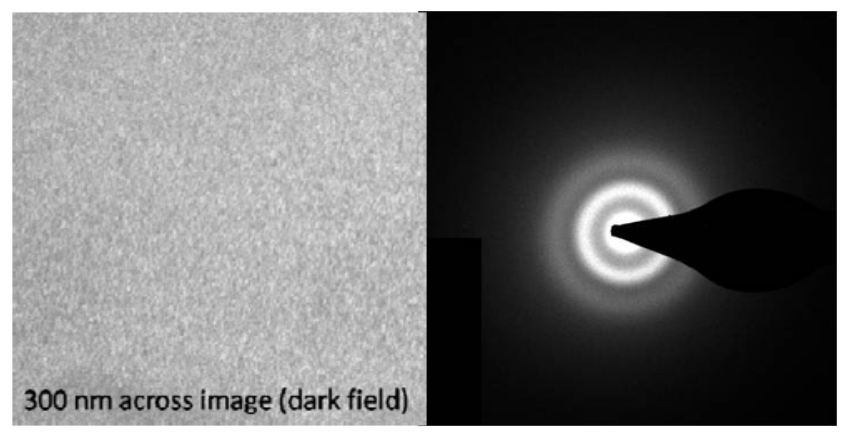

FIG. 1. Low magnification dark field TEM image of ZBLAN (left) and selected area diffraction pattern (right).

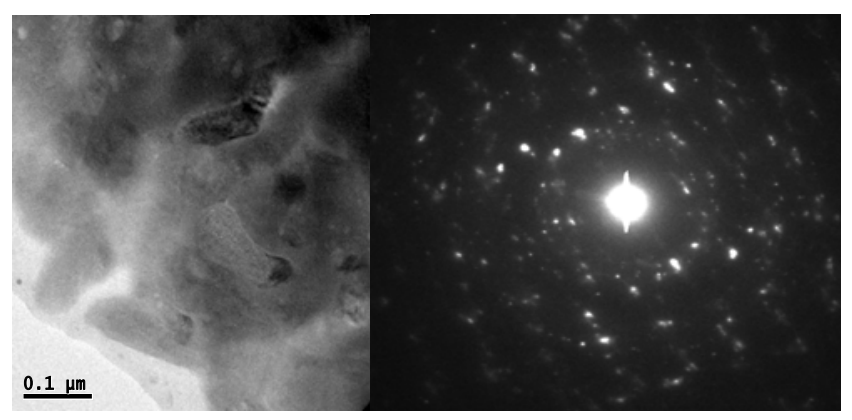

FIG. 2. Bright field TEM image of partially-crystallized ZBLAN.
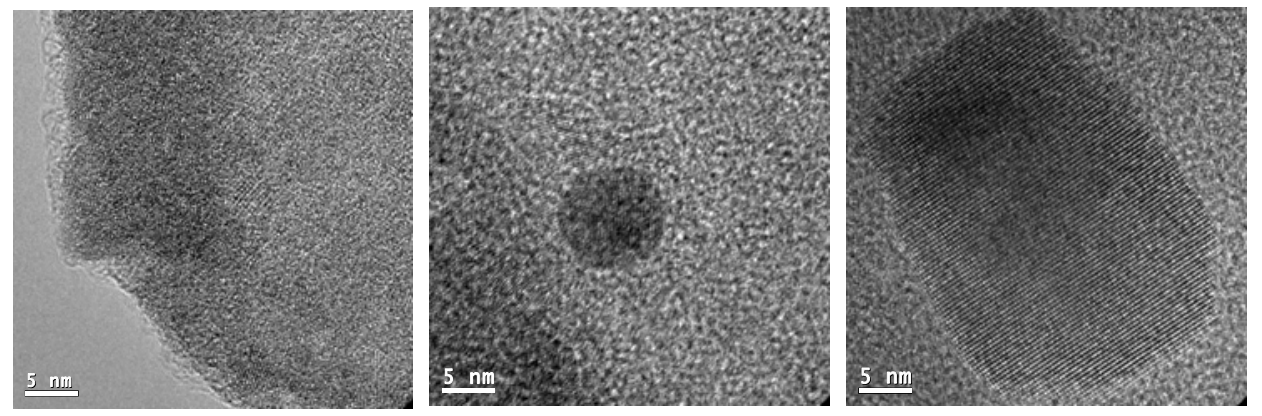

FIG. 3. High resolution TEM of $\mathrm{BaCl}_{2}$ nanoparticals.

[1] J.A. Johnson, S. Schweizer, A.R. Lubinsky, Journal of the American Ceramic Society, 90 (2007) 693-698.

[2] This material is based upon work supported by the National Science Foundation under Grant No. DMR-1001381. The electron microscopy was accomplished at the Electron Microscopy Center for Materials Research at Argonne National Laboratory, a U.S. Department of Energy Office of Science Laboratory operated under Contract No. DE-AC02-06CH11357 by UChicago Argonne, LLC. 